\title{
A New Backoff Algorithm to Guarantee Quality of Service over IEEE 802.11 Wireless Local Area Networks
}

\author{
Kil-Woong Jang \\ Dept. of Applied Science, Korea Maritime University, Busan, Korea \\ jangkw@bada.hhu.ac.kr
}

\begin{abstract}
In this paper, we propose a new backoff algorithm to guarantee quality of service over IEEE 802.11 wireless local area networks. It is designed to carry out the proposed backoff algorithm, which changes the contention window size for backoff time using information of basic service area, such as number of stations. We evaluate the performance of the proposed algorithm using Markov model analysis and compare it with the IEEE 802.11 e backoff algorithm. The numerical results show that the proposed algorithm is able to offer better performance than the conventional backoff algorithm in terms of the throughput.
\end{abstract}

\section{Introduction}

Wireless local area networks (WLANs) provide high bandwidth and real-time multimedia applications for users in a limited geographical area. They are supported by two standards: the IEEE 802.11 standard $[4,5]$ and High Performance Radio LAN (HIPERLAN) Type 2 [6]. In particular, the IEEE 802.11 standard provides two service types: asynchronous and delay bound. The asynchronous service is provided by the distributed coordination function (DCF), which implements the basic access method of the IEEE 802.11 media access control (MAC) protocol. It is also known as the carrier sense multiple access with collision avoidance (CSMA/CA) protocol.

In CSMA/CA, each station seeking access to the medium selects a random time slot within the contention window $(C W)$. The station that selects the shortest random time will gain access for transmission; the others stop their backoff times until the transmission is finished and wait for the remaining time in the following cycle. However, this contention-based MAC protocol is unable to guarantee quality of service (QoS) for time-sensitive traffic. In WLANs, all stations must compete for access to the shared medium. Therefore, the competition may cause longer transmission delay and lower throughput due to collisions.

In this paper, we propose a new backoff algorithm in order to improve the throughput of real-time traffic by resizing the $C W$. Specifically, we develop a Markov model of the IEEE 802.11 DCF protocol. We then use this model to anlayze the properties of the proposed algorithm. 


\section{IEEE 802.11e Backoff Alogrithm}

The IEEE 802.11e standard defines a single coordination function, called the hybrid coordination function ( $\mathrm{HCF}$ ), for the QoS provisioning. The HCF is a function that combines aspects of the $\mathrm{DCF}$ and point coordination function (PCF) to provide the selective handling of frames required for the QoS facility. The HCF uses a contention-based channel access mechanism, referred to as the enhanced DCF (EDCF). A enhanced station (QSTA) operates according to the same general rules defined for EDCF by providing separate output queues. Each queue initiates a EDCF state machine that contends for the wireless medium with $\operatorname{AIFS}[i]$ rather than DIFS, where $i$ is traffic priority and is determined by traffic category (TC) defined as the IEEE 802.11e standard. In addition, it employs a $C W_{\min }[i]$ rather than a $C W_{\min }$ between queues within an QSTA.

The backoff algorithm shall be invoked when a transmitting station infers a failed transmission. To begin the backoff algorithm, the QSTA shall set its backoff timer to a random backoff time using the following equation:

$$
T_{\text {backoff }}[i]=\text { Random }(i) \times \text { SlotTime } .
$$

where Random $(i)$ is a pseudo random integer drawn from a uniform distribution over the interval $[0, C W[i]] . C W[i]$ is an integer within the range of values of the management information base (MIB) attributes a $C W_{\min }[i]$ and a $C W_{\max }[i]$.

To compute the new $C W[i]$ value, denoted $C W_{\text {new }}[i]$, from the old $C W[i]$ value, denoted $C W_{\text {old }}[i]$, in the event of a collision, a station shall choose a value of $C W_{\text {new }}[i]$ that meets the following criterion:

$$
C W_{\text {new }}[i]=\left(\left(C W_{\text {old }}[i]+1\right) \times P F\right)-1 .
$$

where the persistence factor, $P F$, is computed using the procedure described in [5]. The values of $C W_{\min }[i], \operatorname{AIFS}[i]$ and $P F$ are transmitted by an enhanced access point (QAP) using the management frame with the QoS parameter set element.

\section{Proposed Backoff Algorithm}

In this section, we present the proposed backoff algorithm to increase the throughput for time-sensitive traffic over IEEE 802.11e WLANs. Our basic idea is to allow the backoff algorithm to resize the $C W$ according to a number of stations.

Of IEEE 802.11e standard management frames, the management frame with the QBSS load element contains information on the current station population in the QoS basic service set (QBSS). The station count field in the frame indicates the total number of normal stations (STAs) and QSTAs currently associated with this QBSS. Using the station count field of this frame, the QAP can control the $C W$ size under the certain status of transmission.

The proposed algorithm is attempted as follows. We first assume that the traffic is divided into two types: real-time and non-real-time traffic. According to 
the traffic type, the proposed algorithm carries out a retransmission procedure using the different $C W$. We distinguish whether a station can support the QoS or not. If a station is a STA, the backoff procedure is conducted according to the conventional backoff procedure. The set of $C W$ values shall be sequentially ascending integer powers of the value of default $P F$, minus 1 , beginning with a $C W_{\min }$ value and continuing up to and including a $C W_{\max }$ value. In this paper we assume that the value of the default $P F$ is 2 .

If a station is a QSTA, the backoff procedure carries out the proposed algorithm. In the proposed algorithm, for real-time traffic, QAPs set a boundary, called a threshold, $\phi$, to distinguish the $C W$ size under two states of transmission in QBSS: the idle and busy states. If a number of stations, $n$ is lower than $\phi$, it is called an idle state. Otherwise, it is called a busy state.

In an idle state, to reduce the waiting delay during the backoff procedure, a high-priority station with real-time traffic has a smaller $P F$ value than the default PF value. However, if traffic occurs heavily, collisions will be increased because the $C W$ for real-time traffic has a low value. Therefore, the amount of the transmitted frame is reduced due to high collisions. To reduce the occurrence of collisions, in a busy state the $C W$ value is increased as the QAP increases the $P F$ value.

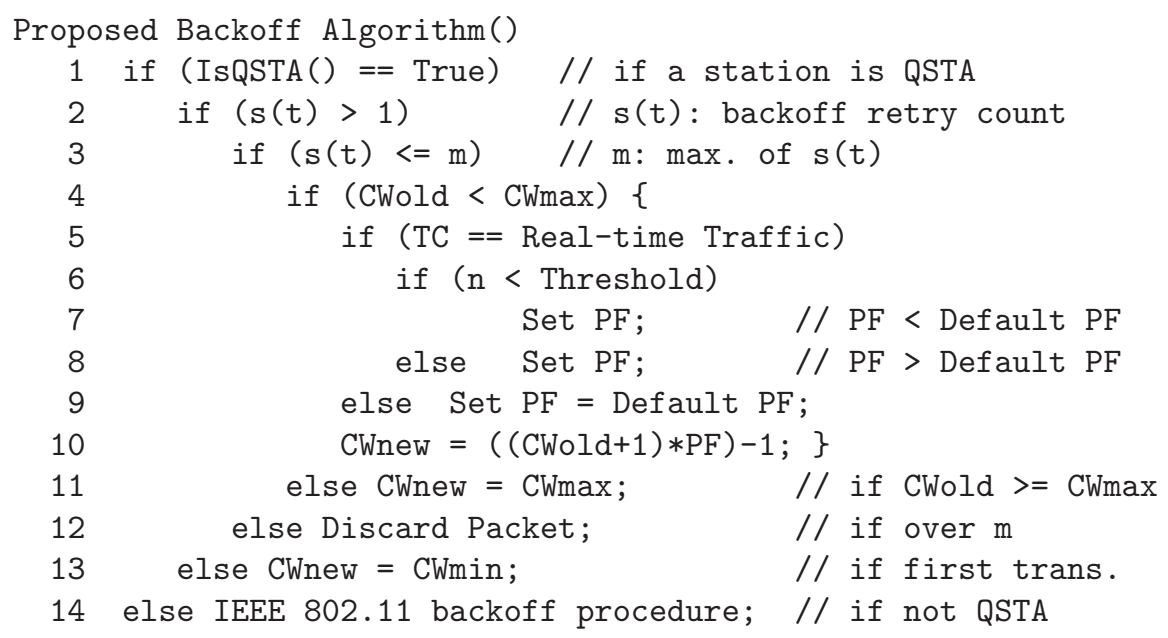

\section{Performance Evaluation}

We present a Markov model to obtain the throughput, $\gamma$. A Markov model for backoff time was proposed in [1-3] to analyze the performance of the IEEE 802.11 protocol that only employs the DCF. In order to accurately analyze the performance of the DBA, we adopt the Markov model proposed in [1-3] and we present the modified Markov model to be suited the proposed algorithm, as shown in Fig. 1. 


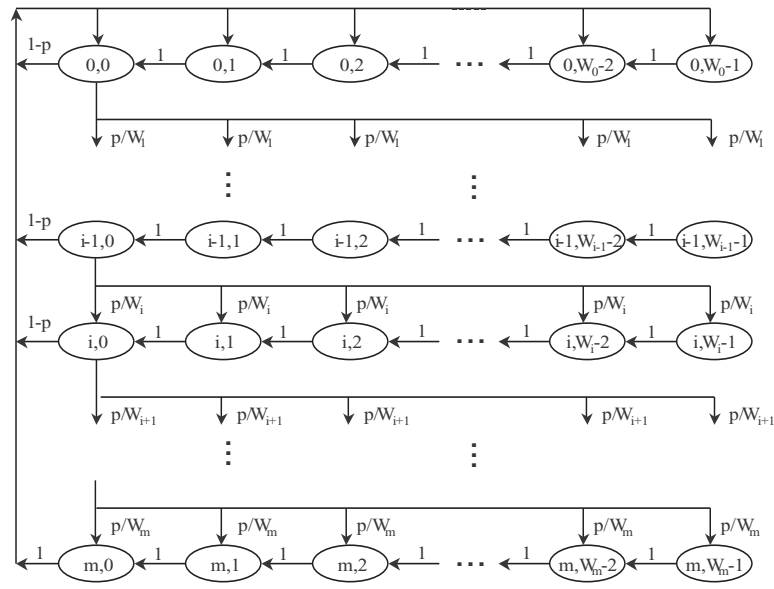

Fig. 1. Markov model for backoff time

We consider a fixed number $n$ of contending stations. Let $s(t)$ be the stochastic process representing the backoff retry count of the station at time $t$ and $c(t)$ be the stochastic process representing the backoff time counter for a station at time $t$. In addition, let $m$ be the maximum value of the backoff retry count. We assume that each frame collides with constant and independent probability $P_{c}$. Once independence is assumed and $P_{c}$ is supposed to be a constant value, the tuple $\{s(t), c(t)\}$ is a discrete-time Markov chain with transition probabilities [1]. In this Markov chain we set $P\left\{i_{1}, j_{1} \mid i_{0}, j_{0}\right\}=P\left\{s(t+1)=i_{1}, c(t+1)=j_{1} \mid\right.$ $\left.s(t)=i_{0}, c(t)=j_{0}\right\}$. To describe the decrement of the backoff time counter, we have

$$
\left\{\begin{array}{l}
P\{i, j \mid i, j+1\}=1 \quad\left(0 \leq i \leq m, \quad 0 \leq j \leq W_{i}-2\right) \\
P\{0, j \mid i, 0\}=1-P_{c} / W_{0} \quad\left(0 \leq i \leq m-1, \quad 0 \leq j \leq W_{0}-1\right) \\
P\{i, j \mid i-1,0\}=P_{c} / W_{i} \quad\left(1 \leq i \leq m, \quad 0 \leq j \leq W_{i}-1\right) \\
P\{0, j \mid m, 0\}=1 / W_{0} \quad\left(0 \leq j \leq W_{m}-1\right)
\end{array} .\right.
$$
have

Here, we let $c_{i, j}$ be the stationary distribution of the Markov chain and we

$$
c_{i, j}=\lim _{t=\infty} P\{s(t)=i, c(t)=j\} \quad\left(1 \leq i \leq m, \quad 0 \leq j \leq W_{i}-1\right) .
$$

Due to the Markov chain regularities, we can obtain a solution for this Markov chain. The solutions are derived as follows:

$$
\begin{gathered}
c_{i, 0}=P_{c} c_{i-1,0} \rightarrow c_{i, 0}=P_{c}^{i} c_{0,0} \quad(1 \leq i \leq m) . \\
c_{i, j}=\frac{W_{i}-j}{W_{j}}\left\{\begin{array}{ll}
\left(1-P_{c}\right) \sum_{k=0}^{m-1} c_{k, 0}+c_{m, 0} & (i=0) \\
P_{c} c_{i-1,0} & (0<i \leq m)
\end{array} .\right.
\end{gathered}
$$


We can obtain the value of $c_{0,0}$ by imposing the normalization condition

$$
1=\sum_{i=0}^{m} \sum_{j=0}^{W_{i}-1} c_{i, j}=c_{0,0} \sum_{i=0}^{m} P_{c}^{i} \frac{W_{i}+1}{2}=\frac{c_{0,0}}{2}\left[W_{0} \sum_{i=0}^{m}\left(P F \cdot P_{c}\right)^{i}+\sum_{i=0}^{m} P_{c}^{i}\right] .
$$

Now, we can obtain $\xi$, which is the probability that a station transmits in a slot time. As any transmission occurs when the backoff size is equal to zero, we have

$$
\xi=\sum_{i=0}^{m} c_{i, 0}=\frac{c_{0,0}}{1-P_{c}} .
$$

Let $P_{t}$ be the probability that there is at least one transmission in a slot time and let $P_{s}$ be the probability that a frame is transmitted successfully. Once $\xi$ is known, $P_{t}$ and $P_{s}$ can be obtained as follows:

$$
\begin{gathered}
P_{t}=1-(1-\xi)^{n} . \\
P_{s}=\frac{n \xi(1-\xi)^{n-1}}{P_{t}}=\frac{n \xi(1-\xi)^{n-1}}{1-(1-\xi)^{n}} .
\end{gathered}
$$

We let $L_{f}$ be the average amount of payload information successfully transmitted in a slot time and $L_{s}$ be the length of a renewal interval. Finally, we can determine $\gamma$, defined by

$$
\gamma=\frac{L_{f}}{L_{s}}=\frac{P_{s} P_{t} L_{p}}{\left(1-P_{t}\right) S_{t}+P_{s} P_{t} T_{s}+\left(1-P_{s}\right) P_{t} T_{c}} .
$$

where $L_{p}$ is the average frame length and $S_{t}$ is the size of a slot time. Moreover, $T_{s}$ is the average time that the channel is sensed busy due to a successful transmission and $T_{c}$ is the average time that channel is sensed busy by the stations during a collision. We assumed for simplicity that all the stations use the RTS/CTS access method for all the transmitted frames over the IEEE 802.11 networks. In such a case collisions can occur only on RTS frames. We obtained values for the throughput under various system parameters. The analysis results were obtained using the OFDM system parameters.

In the proposed algorithm, according to the $P F$ value during the backoff procedure, the throughput can be affected by the $P F$. According to the traffic type, we applied the $P F$ value to the $C W$ size in the above equations. The $P F$ values for the real-time in an idle and a busy state are denoted by $w_{1}$ and $w_{2}$, respectively. Additionally, the $P F$ value for the non-real-time traffic in a busy state is denoted by $w_{3}$. We considered two cases: case $1\left(w_{1}=1, w_{2}=3\right.$ and $\left.w_{3}=2\right)$ and case $2\left(w_{1}=1.5, w_{2}=2.5\right.$ and $\left.w_{3}=2\right)$.

Fig. 2 shows the throughput theoretically achievable by the backoff algorithm in both the cases of the IEEE 802.11e and proposed algorithm. From this figure, we can see that the proposed algorithm performs better than the IEEE $802.11 \mathrm{e}$ under most traffic loads. In Fig 2(a) and (b), we changed the backoff algorithm at $\phi=6$ and $\phi=7$, respectively. Around thess points, the performance of the proposed algorithm is partially lower than the IEEE 802.11e. However, we can see that the proposed algorithm using threshold and $P F$ values performs better than the conventional backoff algorithm under heavy traffic loads. 

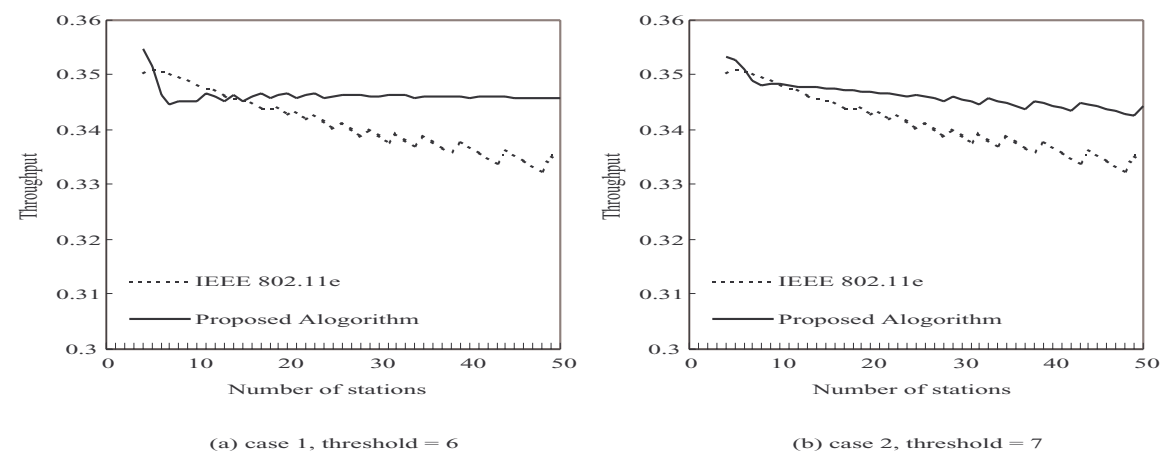

Fig. 2. Throughput under different numbers of stations

\section{Conclusions}

In this paper, we proposed a new backoff algorithm to enhance the throughput in IEEE 802.11e WLANs. Our basic idea is to resize the contention window under a pre-defined threshold in QBSS. We developed a Markov model to compare the proposed algorithm and the IEEE 802.11e for backoff time. We then evaluated the throughput for the proposed algorithm and IEEE 802.11e backoff algorithm. Numerical results demonstrate that the proposed algorithm performs better than the conventional IEEE 802.11e standard with EDCF in WLAN.

\section{References}

1. G. Bianchi.: Performance analysis of the IEEE 802.11 distributed coordination function. IEEE J. Select. Areas Commun., vol. 18, no. 3, Mar. (2000) 535-547

2. S. T. Sheu, T. F. Sheu.: A bandwidth allocation/sharing/extension protocol for multimedia over IEEE 802.11 ad hoc wireless LANs. IEEE J. Select. Areas Commun., vol. 19, no. 10, Oct. (2001) 2065-2080

3. H. Wu, S. Cheng, Y. Peng, K. Long and J. Ma .: IEEE 802.11 Distributed coordination function(DCF): Analysis and enhancement. Proc. ICC 2002, no. 1, Apr. (2002) 605-609

4. IEEE Standard for Wireless Medium Access Control and Physical Layer Specifications, Aug. (1999)

5. IEEE Std 802.11e/D2.0, Draft Supplement to Part 11: Wireless Medium Access Control and Physical Layer Specifications, Medium Access Control Enhancements for Quality of Service, Nov. (2001)

6. Broadband Radio Access Networks HIPERLAN Type 2, Apr. (2000) 